Investigación Descriptiva

Pensar en Movimiento:

Revista de Ciencias del Ejercicio y la Salud ISSN 1659-4436

Vol. 15, No.2, pp. $1-21$

Abre el $1^{\circ}$ de julio, cierre al 31 de diciembre, 2017

\title{
CONSTRUCCIÓN DEL REGISTRO DE OBSERVACIÓN PARA EL ANÁLISIS DEL MOVIMIENTO FUNDAMENTADO EN LA TEORÍA DE LABAN ${ }^{1}$
}

\section{DEVELOPMENT OF AN OBSERVATION INSTRUMENT FOR RECORDING MOVEMENT ANALYSIS BASED ON LABAN'S THEORY}

\section{CONSTRUÇÃO DO REGISTRO DE OBSERVAÇÃO PARA A ANÁLISE DO MOVIMENTO FUNDAMENTADO NA TEORÍA DE LABAN}

Silvia Barnet-López, Dra. 2,3(B,C,D,E); Mar Arbonés-García, Lic. 3(B,D,E); Susana

Pérez-Testor, Dra. 2,3(B,C,E) y Myriam Guerra-Balic, Dra. 2(B,D,E) silviabl0@blanuqerna.url.edu

2Facultat de Psicología, Ciencias de l'Educació i de l'Esport Blanquerna, Universitat Ramon Llull, Barcelona, España

3Universitat Autonoma de Barcelona, Barcelona, España

\author{
Envío original: 16/12/2016; reenviado: 01/10/2017; \\ aceptado: 06/10/2017; publicado: 22/12/2017.
}

Doi: http://dx.doi.org/10.15517/pensarmov.v15i2.27334

\section{RESUMEN}

Barnet-López, S., Arbonés-García, M., Pérez-Testor, S. y Guerra-Balic., M. (2017). Construcción del registro de observación para el análisis del movimiento fundamentado en la teoría de Laban. Pensar en Movimiento: Revista de Ciencias del Ejercicio y la Salud, 15(2), 1-21. En este artículo se describe el proceso de construcción de un Registro de Observación inspirado en el Análisis del Movimiento de Laban (ROAM) en personas con Discapacidad Intelectual (DI). La aplicación del ROAM permite obtener una medida objetiva y estandarizada sobre las tendencias generales de movimiento de la persona, además de poder utilizarse en observaciones pre y post intervenciones y así conocer los cambios de movimiento del individuo. El del ROAM se desarrolló en varias fases, en la primera de las cuales se identificaron

\footnotetext{
$1 \quad$ El presente manuscrito forma parte del proyecto final del Máster en Danza Movimiento Terapia (Universidad Autónoma de Barcelona, realizado por las dos primeras autoras, y que puede ser consultado en el sitio web https://ddd.uab.cat/pub/trerecpro/2015/hdl_2072_253714/Arbones_y_BarnetTesinaDMT 2015.pdf).
} 
los elementos básicos para el registro y se realizaron diversas versiones del ROAM mediante la técnica de jueces expertos. En las fases posteriores se realizó el estudio exploratorio, utilizando el registro para evaluar dos sesiones de danza movimiento terapia en un grupo de adultos con DI. Los resultados muestran que el ROAM presenta unos buenos índices de validez de contenido y de fiabilidad inter e intra observadores (0.72), por tanto, el ROAM permitió analizar las características generales del movimiento de las personas con DI que participaron en el estudio exploratorio.

Palabras claves: registro de observación; análisis del movimiento.

\section{ABSTRACT}

Barnet-López, S., Arbonés-García, M., Pérez-Testor, S. \& Guerra-Balic., M. (2017). Development of an Observation Instrument for Recording Movement Analysis based on Laban's Theory. Pensar en Movimiento: Revista de Ciencias del Ejercicio y la Salud, 15(2), 1-21. This paper describes the development process of an observation record inspired in Laban's Movement Analysis (ROAM) for Intellectually Disabled (ID) people. An objective and standardized measurement of a person's general movement tendencies can be obtained from ROAM, in addition to making observations before and after an intervention, therefore learning about movement changes of an individual. The design of ROAM was developed in several stages: for the first one, basic elements were identified for the record and different versions of ROAM were created using the expert judges technique. Later stages involved an exploratory study, where two sessions of movement-dance therapy were evaluated in a group of ID adults using the record. Our results show that ROAM exhibits good indexes for content validity as well as intra- and inter-observer reliability (0.72). Thus, we conclude that ROAM allowed us to analyze the general movement characteristics of intellectually disabled individuals participating in this exploratory study.

Key words: observation log; motion analysis.

\section{RESUMO}

Barnet-López, S., Arbonés-García, M., Pérez-Testor, S. \& Guerra-Balic., M. (2017). Construção do registro de observação para a análise do movimento fundamentado na teoría de Laban. Pensar en Movimiento: Revista de Ciencias del Ejercicio y la Salud, 15(2), 1-21. Neste artigo se descreve o processo de construção de um Registro de Observação inspirado na Análise do Movimento de Laban (ROAM) em pessoas com Deficiência Intelectual (DI). A aplicação do ROAM permite obter uma medida objetiva e padronizada sobre as tendências gerais de movimento da pessoa, além de ser possível sua utilização em observações pré e pós intervenções e assim conhecer as mudanças de movimento do indivíduo. O desenho do ROAM foi desenvolvido em várias fases, na primeira das quais se identificaram os elementos básicos para 0 registro e realizaram diversas versões de ROAM através da técnica de juízes peritos. Nas fases posteriores foi realizado o estudo exploratório, utilizando o registro para avaliar duas sessões de dança movimento terapia em um grupo de adultos com DI. Os resultados mostram que o ROAM apresenta alguns bons índices de validade de conteúdo e de fiabilidade inter e intraobservadores $(0,72)$, portanto, o ROAM permitiu analisar as características gerais do movimento das pessoas com DI que participaram no estudo exploratório.

Palavras-chave: registro de observação; análise do movimento. 
Este estudio se centra en el movimiento del cuerpo y la conducta no verbal a partir del análisis del movimiento propuesto por Laban (1978). Las conclusiones de los estudios que han utilizado metodología observacional, han demostrado que existe una relación entre los movimientos del cuerpo y los distintos procesos psicológicos. Aunque predominan las investigaciones centradas en los elementos donde interviene la expresión verbal de la persona, en el caso de estudios centrados en conductas no verbales como son los gestos, expresión facial, utilización del espacio u otros elementos vinculados con el movimiento y la expresión corporal, se ha podido ver cómo el cuerpo y sus acciones son una importante fuente de información (Anguera, 1989).

En el análisis del movimiento, la aplicación de un instrumento válido y confiable de observación proporcionará rigurosidad y objetividad a los estudios de este ámbito (Bakeman, Gottman, Portal, Argilaga y Villaseñor, 1989).

\section{Teoría de Análisis del Movimiento de Laban}

La teoría de Laban surge del estudio y análisis de los patrones de movimiento, con el fin de crear un vocabulario con significado propio, perpetuo al paso del tiempo. Así se establecieron las bases de la relación cuerpo-mente y la intencionalidad de las acciones, de las cuales nace el Análisis del Movimiento de Laban (AML) (Bishko, 2014).

El AML es un sistema creado y empleado para la observación, descripción, anotación e interpretación del movimiento de las personas, el cual permite la obtención de un perfil del movimiento (Foroud y Whishaw, 2006). El movimiento de la persona se encuentra en una evolución continua, con intencionalidad y regido por un orden. El AML lo estructura y aborda sus diferentes niveles para analizar su significado y poder ser entendido (Moore y Yamamoto, 1988).

Laban identificó tres niveles del movimiento, tres postulados que fundamentan la base del análisis a realizar: la mente se sumerge en las emociones e ideas; la objetividad externa del observador; así como la vivencia corporal de la persona, con lo que su observación y análisis tiene que enfocarse desde la misma perspectiva (Bishko, 2014). De este modo, el AML describe patrones que son consistentes para un individuo y que le diferencian de los otros, y permite identificar los patrones comunes dentro de un mismo tipo de población o grupo de trabajo. Por lo tanto, proporciona un análisis objetivo de la calidad del movimiento de todo tipo de población (Laban, 1978). La calidad del movimiento es aquella cualidad expresiva que la persona le da al movimiento. El individuo administra la energía del movimiento, el cual se manifiesta en diferentes grados, según la combinación de espacio, tiempo, intensidad y fluidez de este (Schinca, 2010).

Una persona puede necesitar usar todo el espacio disponible para moverse, con matices abruptos y de súbitos cambios de dirección; mientras que otra emplea solo el espacio que ocupa su cuerpo, con movimientos ligeros y sostenidos, no siempre producido con la misma calidad. La riqueza del movimiento está en poder combinar diferentes calidades y adaptarnos a cada situación. En personas con dificultades cognitivas o de expresión se observa un repertorio de movimiento limitado, y por lo 
tanto podemos observar cómo algunas de estas calidades no aparecen en los movimientos de la persona. El ser humano puede cambiar o evolucionar, consciente o inconscientemente, así como adaptarse a lo nuevo o diferente (Fischman, 2001; Laban, 1987). Así pues, el AML integra los elementos cuerpo, espacio, esfuerzos, relaciones y acciones (Zhao, 2001; Laban 1987).

Cuerpo. El cuerpo incluye los aspectos estructurales del movimiento, como son la continuidad de la acción de una parte a la otra; la coordinación y la postura corporal que surgen de los gestos que se realizan; así como la funcionalidad del movimiento, siendo el medio a través del cual manifestamos aquello que queremos expresar de forma consciente e inconsciente (Bishko, 2014). Para entender y describir el movimiento corporal, observamos las zonas del cuerpo conectadas entre sí, la influencia de las unas en las otras, así como su orden y estructura (Ros, 2009). El cuerpo es una estructura simétrica compuesta por características estructurales y físicas, así como la coordinación entre sus diferentes partes. Incluye parte física y orgánica, lo que se establece como la base del movimiento (Longstaff, 2007).

Espacio. El espacio engloba el análisis del contexto del movimiento corporal, siendo diversos los aspectos que debemos observar. Las estructuras espaciales que realiza la persona en movimiento; los caminos que dibuja; así como las líneas de tensión en el espacio, las cuales representan el recorrido del cuerpo o de una de sus partes (Ros, 2009). Laban, en el análisis del espacio, estableció el concepto de la esfera del movimiento (kinesfera), la cual es el espacio que rodea al cuerpo, demarcado por lo que ocupa la persona con las extremidades extendidas. Para visualizarlo, imaginamos al sujeto dentro de una esfera, capaz de acceder con sus extremidades a cualquiera de sus puntos (Laban, 1978), relacionándose también con los demás y el entorno (Ridocci, 2009).

Esfuerzos. Los esfuerzos son "factores de movilidad hacia los cuales la persona que se mueve adopta una actitud definida" (Laban, 1987, p.131); sus combinaciones son el resultado de las acciones realizadas. Todo individuo sano tiene la capacidad para poder desarrollarlos en su globalidad (Laban, 1978). Con los esfuerzos podemos trabajar diferentes elementos, como son la actitud de liderazgo, el contacto visual, la capacidad para ver y atender al otro y la capacidad de reacción. Estos elementos impulsan el sentimiento de pertenencia hacia el grupo. Si trabajamos en sesiones grupales, aumentan las posibilidades de relación con el otro, lo que facilita y promueve el establecimiento de diálogos en movimiento (Laban, 1978).

Acciones. Las acciones básicas son las combinaciones de los esfuerzos que realiza la persona, encontrando en cada acción esfuerzos que destacan. Así pues, al identificar la acción, se podrá determinar los esfuerzos presentes o el que predomina en esa acción en concreto. Laban (1978) determina la existencia de ocho acciones básicas (ver Tabla 1). 
Tabla 1

\begin{tabular}{|c|c|c|c|}
\hline Las & acciones & Laban & $(197$ \\
\hline Acciones & Descripción & Ejemplos & Element \\
\hline Presionar & $\begin{array}{l}\text { Fuerte resistencia y empuje } \\
\text { unidireccional (peso-espacio). } \\
\text { Sostener la acción durante un } \\
\text { periodo de tiempo determinado } \\
\text { ("abandonarse"- tiempo). }\end{array}$ & $\begin{array}{l}\text { Intentar mover una } \\
\text { pared utilizando una o } \\
\text { más partes del cuerpo. }\end{array}$ & $\begin{array}{l}\text { Firme } \\
\text { Directa } \\
\text { Sostenida }\end{array}$ \\
\hline $\begin{array}{l}\text { Dar latigazos } \\
\text { leves }\end{array}$ & $\begin{array}{l}\text { Aflojar la tensión muscular y lograr } \\
\text { una sensación de ligereza. } \\
\text { Abandonar el tirón unidireccional y } \\
\text { rendirse al movimiento continuo } \\
\text { ("abandonarse" al peso-espacio). } \\
\text { Acelerar la acción, ocurre en un } \\
\text { periodo corto de tiempo ("luchar } \\
\text { contra"-tiempo). }\end{array}$ & $\begin{array}{l}\text { Movimientos de tocar el } \\
\text { piano con los dedos. }\end{array}$ & $\begin{array}{l}\text { Ligera } \\
\text { Flexible } \\
\text { Súbita }\end{array}$ \\
\hline $\begin{array}{l}\text { Dar } \\
\text { puñetazos } 0 \\
\text { arremeter }\end{array}$ & $\begin{array}{l}\text { Desarrolla un control de esfuerzos } \\
\text { ("luchar contra" -peso-espacio- } \\
\text { tiempo) y la elasticidad } \\
\text { de las tensiones opuestas, directas y } \\
\text { rápidas. }\end{array}$ & $\begin{array}{l}\text { Dar una patada o golpe } \\
\text { en el aire. }\end{array}$ & $\begin{array}{l}\text { Súbita } \\
\text { Directa } \\
\text { Firme }\end{array}$ \\
\hline $\begin{array}{l}\text { Flotar } \\
\text { o volar }\end{array}$ & $\begin{array}{l}\text { Control de esfuerzos (“abandonarse } \\
\text { al"-tiempo-peso-espacio) y las } \\
\text { tensiones opuestas leves y } \\
\text { multilaterales, producen un tipo de } \\
\text { sustentación suave. }\end{array}$ & $\begin{array}{l}\text { Revolotear en el aire en } \\
\text { un salto. }\end{array}$ & $\begin{array}{l}\text { Sostenida } \\
\text { Flexible } \\
\text { Ligera }\end{array}$ \\
\hline Retorcerse & $\begin{array}{l}\text { Control de esfuerzos y la firmeza } \\
\text { continua de las tensiones opuestas, } \\
\text { produce un tipo especial de } \\
\text { flexibilidad sostenida } \\
\text { ("abandonarse"-tiempo-espacio y } \\
\text { "luchar"-peso). }\end{array}$ & $\begin{array}{l}\text { Escurrir un trapo con las } \\
\text { manos. }\end{array}$ & $\begin{array}{l}\text { Flexible } \\
\text { Sostenida } \\
\text { Firme }\end{array}$ \\
\hline $\begin{array}{l}\text { Dar toques } \\
\text { ligeros }\end{array}$ & $\begin{array}{l}\text { Cierta relajación y sensación de } \\
\text { ingravidez ("lucha"-tiempo-espacio y } \\
\text { "abandonarse"-peso). Sensación } \\
\text { directa y repentina (rebote elástico). }\end{array}$ & $\begin{array}{l}\text { Los dedos al escribir en } \\
\text { el ordenador. }\end{array}$ & $\begin{array}{l}\text { Directa } \\
\text { Súbita } \\
\text { Ligera }\end{array}$ \\
\hline $\begin{array}{l}\text { Hendir } \\
\text { el aire }\end{array}$ & $\begin{array}{l}\text { Ágil ajuste de tensiones opuestas, } \\
\text { fuertes y multilaterales, produce } \\
\text { fluidez flexible ("lucha"-peso-tiempo y } \\
\text { "abandonarse"-espacio). }\end{array}$ & $\begin{array}{l}\text { Un grand jete en danza } \\
\text { clásica (saltar). }\end{array}$ & $\begin{array}{l}\text { Súbita } \\
\text { Firme } \\
\text { Flexible }\end{array}$ \\
\hline Deslizarse & $\begin{array}{l}\text { Control de esfuerzo mayor que en } \\
\text { inclinarse y estirarse suavemente } \\
\text { ("abandonarse" peso-tiempo y } \\
\text { "lucha"-espacio). }\end{array}$ & $\begin{array}{l}\text { Hacer el gusano por el } \\
\text { suelo. }\end{array}$ & $\begin{array}{l}\text { Sostenida } \\
\text { Ligera } \\
\text { Directa }\end{array}$ \\
\hline
\end{tabular}

Fuente: elaboración propia, con base en Teoría de Laban.

Por una parte, las ocho acciones básicas conjuran una clasificación global por categorías que permite analizar el movimiento por secuencias, así como ver de una 
forma más clara las cualidades del movimiento. Por la otra, se acostumbra observar una combinación de las acciones en el movimiento de una persona (Laban, 1978).

\section{Metodología observacional}

La observación está presente desde los inicios de las investigaciones, siendo uno de los primeros métodos utilizados para la recogida de datos (Anguera, 1989). Nos centramos en una observación científica y no en una observación superficial, por ese motivo es importante que sea lo más objetiva posible. Para ello, será relevante determinar la unidad de análisis con una definición útil para poder aplicar en el estudio, pero con una cierta flexibilidad imprescindible para poder abordar la complejidad y la riqueza del fenómeno de estudio (Sarriá y Brioso, 1999).

La metodología observacional ha experimentado un crecimiento constante. Tal y como afirman Anguera y Hernández-Mendo (2014), es una herramienta que se utiliza de forma exitosa dentro del conocimiento de la psicología del deporte. Para ello será importante la rigurosidad en la que haya sido construido el instrumento y que el observador tenga los conocimientos necesarios para realizar una observación objetiva, fiable y profesional (Anguera 1989, 1999).

Centrándonos en el registro observacional, uno de los elementos que conforman la metodología observacional, será clave considerar la opinión de expertos investigadores para poder determinar las categorías de análisis. Por lo tanto, se recomienda buscar un equilibrio entre la realización de un registro preciso de las variables relevantes para nuestro campo de estudio y la información significativa no esperada que este puede aportarnos (Anguera, 1989).

Así pues, con el propósito de obtener una buena codificación, será importante tener una base teórica consistente para ofrecer al investigador una lente que le permitirá contemplar su mundo, su objeto y variables del estudio (Bakeman, et al., 1989). El sistema empleado debería separar el comportamiento en categorías de características y criterios específicos, usando un vocabulario concreto para entender y elaborar la realidad observada en un registro enmarcado (Izquierdo y Anguera, 2000).

La observación y el análisis del movimiento están muy presentes dentro de disciplinas como la danza, la expresión corporal o la danza movimiento terapia (DMT). Estas disciplinas consideran el movimiento como un vehículo para la expresión de sentimientos y emociones del pensamiento, así como un mecanismo mediante el cual las sociedades trasladan modos de comportamiento (Fischman, 2001; Vella y Torres, 2012). Además, nos permiten trabajar con todo tipo de población, incluyendo a personas que presentan limitaciones en la expresión verbal, a nivel cognitivo, de comprensión o a nivel de autonomía respecto a los demás. Los acercamientos de carácter creativo dan espacio a la expresión más libre a través del movimiento del sujeto, sin forzar un razonamiento previo (Hurley, Tomasulo y Fadt, 1998).

En el caso de población con dificultades en la expresión oral o con otro tipo de dificultades, se deberá tener en cuenta las posibles dificultades que pueden presentar a la hora de obtener una evaluación válida, así como de aplicar un tipo de evaluación poco intrusiva (Anguera, 1989; Paredes, 2010). Hasta el momento no se ha encontrado ningún instrumento que evalúe los cambios de movimiento teniendo presente este tipo de población (Barnet-Lopez, Pérez-Testor, Arbonés-Garcia y Guerra-Balic, 2015). 


\section{Objetivo del estudio}

El objetivo principal de este estudio fue construir un registro de observación para analizar el movimiento general de la persona, fundamentado en la teoría del AML, el cual toma en cuenta a las personas que pueden presentar limitaciones a nivel físico, cognitivo, emocional o relacional.

Los objetivos específicos del estudio fueron los siguientes: identificar los elementos básicos del AML para proponer una hoja de observación, presentar las diferentes fases de construcción del instrumento y realizar un estudio exploratorio para conocer el grado de fiabilidad intra e inter observadores en la aplicación del instrumento con personas con DI.

\section{METODOLOGÍA}

Procedimiento. Este estudio se desarrolló en 11 fases (ver Tabla 2). En primer lugar se presenta de la fase 1 hasta la fase 8 , las cuales están vinculadas con la elaboración y construcción del instrumento, además de la aplicación de la técnica de los jueces expertos para aportar validez en el contenido del registro. Durante este proceso se realizaron cinco versiones del instrumento, con la obtención de la última versión en la fase 8 . La fase 1 corresponde a la revisión bibliográfica para el diseño del instrumento; las bases de datos consultadas fueron SportDiscus, PudMed, Web of Science, Google Scholar, Dialnet, Psycinfo. La fase 2 se centró en profundizar en las teorías de expertos en metodología observacional para la construcción del registro. Se identificaron, categorizaron y definieron los elementos principales según la fundamentación de la teoría de Laban (1978, 1987).

En las fases 3, 4, 5, 6 y 7 se realizaron cuatro versiones del registro aplicando la técnica de jueces expertos (ver Tabla 2). Se solicitó la colaboración de los jueces vía e-mail y enviando la información referente al proyecto. Tras su aceptación, se les adjuntó la propuesta del registro y se les se solicitó que valorasen diferentes aspectos sobre la información inicial, la escala de medida, las categorías y los ítems del cuestionario, así como que brindaran una valoración global del mismo. Las opiniones fueron consideradas para la reelaboración de las nuevas versiones del registro si se repetía la misma idea en más de uno de los expertos.

En la revisión de la cuarta versión, fase 7 , se aplicaron las últimas consideraciones de los expertos y además se contó con el asesoramiento de un experto en la Teoría del AML, el cual propuso pequeños cambios relacionados con la puntuación del enraizamiento y el equilibrio, así como incluir dentro de forma del cuerpo la opción de "asimétrica". Así pues, finalmente, en la fase 8 , se realizó la última versión del registro obteniendo la aceptación de todos los jueces expertos, obteniendo la versión final del registro.

Una vez creado el instrumento, se procedió al estudio exploratorio (fase 9) para conocer el grado de fiabilidad intra e inter observadores en la aplicación del registro con personas con DI. Para completar el estudio se desarrollaron las fases 10 y 11, donde se analizaron los resultados, discusión y conclusiones finales. En la Tabla 2 encontramos un resumen de las fases del estudio. 
Tabla 2

Fases del estudio

\begin{tabular}{ll}
\hline Fase & Actividades realizadas \\
\hline Fase 1 & $\begin{array}{l}\text { Revisión bibliográfica, clarificación de conceptos, marco teórico y } \\
\text { metodología. }\end{array}$
\end{tabular}

Fase 2 Identificación de los elementos considerados principales para el registro:

- Definir de forma precisa cada uno de los elementos.

- Identificar los elementos de categorización.

- Determinar el número de dimensiones.

- Determinar las características de la observación.

Fase 3 Primera versión del registro de observación:

- Elaboración de la primera versión del registro.

- Revisión: técnica de jueces expertos. Cuatro jueces: dos expertos del AML y dos expertos en la DMT.

Fase 4 Segunda versión del registro de observación:

- Elaboración de la segunda versión del registro.

- Revisión: técnica de jueces expertos.

- Reunión con un asesor experto en el AML para realizar de la forma más rigurosa los cambios propuestos por los jueces expertos.

Fase 5 Tercera versión del registro de observación:

- Elaboración de la tercera versión del registro.

- Revisión: técnica de jueces expertos.

Fase 6 Cuarta versión del registro de observación:

- Elaboración de la cuarta versión del registro.

- Revisión: técnica de jueces expertos. Se incluye la revisión de un quinto juez experto en la Teoría de Laban y en el AML.

Fase 7 Reunión con un experto de metodología y estadística.

Fase 8 Versión definitiva del registro de observación. Se obtiene el Registro de Observación de Análisis del Movimiento (ROAM).

Fase 9 Estudio exploratorio. Aplicación del ROAM: 6 hojas de registro evaluando sesiones de una intervención de danza basada en la DMT en adultos con discapacidad intelectual. Cada sesión fue evaluada por dos observadores expertos en psicología y DMT de forma individual.

Fase 10 Análisis de los datos:

- Validez: se realizó una revisión de los conceptos de la Teoría de Laban y la definición de cada una de las variables. Además, se aplicó la técnica de jueces expertos (ver Fase 3, 4, 5, 6, 7 y 8).

- Fiabilidad: se calculó el índice Kappa de Cohen (inter-observador y intra-observador).

Fase 11 Discusión de los resultados y conclusiones finales.

Fuente: Elaboración propia.

Este estudio ha sido respetuoso con los principios éticos que rigen cualquier investigación realizada con seres humanos, de acuerdo con la declaración de Helsinki. Los participantes fueron informados de las características y finalidades del estudio, así como sus familiares o tutores legales, quienes posteriormente firmaron el consentimiento informado (Amaro, Marrero, Valencia, Blanca y Moynelo, 1996).

Estudio exploratorio: Aplicación del registro. Se realizó un estudio exploratorio utilizando una selección de muestra por conveniencia. Los participantes 
realizaron una intervención grupal en DMT durante 16 sesiones dos veces a la semana, cada sesión era de 60 minutos. Se evaluaron la sesión cuatro y la sesión dieciséis. De este modo, se dejó un período de adaptación a la cámara de video de tres sesiones, para evitar el efecto reactividad. Las sesiones fueron registradas con una cámara de video SONY DCR-SR75E. Posteriormente, los videos fueron analizados de forma individual por dos observadores (observador 1 y observador 2). Finalmente, se introdujeron los resultados de las puntuaciones en el programa estadístico SPSS para obtener el índice de Kappa de Cohen (Fase 9, ver Tabla 2).

Con la finalidad de conocer hasta qué punto dos observadores independientes interpretan de la misma manera las dimensiones que conforman el ROAM, aportando una primera evidencia de fiabilidad del instrumento, se aplicó el índice de Kappa de Cohen. Esta es una medida estadística muy usada en el establecimiento del grado de concordancia entre dos críticos, observadores o jueces, pudiendo ser empleada con cualquier tipo de variable, ya sean cuantitativas o cualitativas (Muñiz, 1992).

A continuación, se exponen las características principales de los jueces expertos durante la elaboración del ROAM, de las sesiones observadas y de los participantes observados.

Jueces expertos. Los jueces expertos participantes en el proceso de construcción del ROAM fueron profesionales del ámbito de la psicología clínica e investigadores activos. Dos de ellos son especialistas en DMT, en el ámbito docente y terapéutico. Los dos restantes son especialistas en el AML y en la metodología observacional. Finalmente, el último juez experto participante es especialista en la Teoría de Laban y el AML, dedicado a la investigación y docencia en dicho ámbito, siendo un referente en cuanto a su conocimiento.

Participantes. Los participantes iniciales del estudio fueron un grupo DMT de personas adultas mayores con DI que participaban en un taller ocupacional diurno de 9 a 17 horas, en la ciudad de Girona (Catalunya), compuesto por siete personas. Tenían reconocido el grado severo de discapacidad (> 76\% de discapacidad) (Real Decreto 1971/1999, núm. 22, 2000), el cual clasifica según el porcentaje de discapacidad global de la persona (física, intelectual y/o sensorial). Se aplicaron los siguientes criterios de inclusión: haber realizado un mínimo de 12 sesiones dentro de la intervención de danza de 16 sesiones totales. Los participantes finales para este estudio fueron tres, dos mujeres de 45 y 49 años y un hombre de 48 años.

Sesiones observadas. Los participantes realizaron sesiones grupales en movimiento, desarrollándose a través de la danza basada en la DMT. Todas las sesiones realizadas seguían una misma estructura de cuatro fases: check-in, calentamiento, transición-proceso, check-out. Según la estructura de Chace (1953), ampliamente utilizada en la DMT.

Durante las sesiones se trabajaron los siguientes elementos: el esquema corporal, favoreciendo a una mayor consciencia corporal; los diferentes registros de movimiento, facilitando la ampliación de su repertorio de movimiento; la comunicación, favoreciendo la relación con el otro; el uso del espacio, facilitando una mayor utilización del mismo; explorar nuevas propuestas de movimiento, fomentando 
momentos de improvisación. De este modo, se trabajaban diferentes propuestas en movimiento en función del objetivo que se quería destacar en la sesión. Destacaba el trabajo a través de los ritmos (percusión corporal) y elementos creativos, mediante ejercicios de expresión corporal.

Las sesiones observadas fueron analizadas a través del ROAM por dos observadores, quienes eran psicólogos y DMT activos, tanto en la práctica clínica como en el ámbito de la investigación. Los observadores hicieron el análisis de todas las sesiones de forma independiente para posteriormente introducir sus respuestas para realizar el análisis estadístico. Los profesionales que realizaron las observaciones no formaban parte de los profesionales que configuraron el grupo de jueces expertos.

Análisis estadístico. En cuanto a la validez de contenido, se utilizó la técnica de jueces expertos, cuyas respuestas fueron analizadas. Con el fin de extraer los elementos a modificar o a conservar de cada una de las versiones del registro, se aplicó un análisis de contenido de las respuestas escritas por cada uno de los jueces en cada una de las versiones del instrumento, hasta obtener la versión definitiva, la cual se presenta en los resultados. Esta versión obtuvo la aprobación de todos sus ítems de forma unánime por parte de todos los jueces.

Para conocer la fiabilidad intra e inter observador los cálculos estadísticos se realizaron mediante el software Statistical Package for Social Sciences (versión 21. para Windows, SPSS Inc., Chicago, IL, USA). Se calculó la fiabilidad con el índice Kappa de Cohen, para relacionar el acuerdo que existe entre los observadores, por equivalencia o concordancia (Landis y Koch, 1977 y Hoehler, 2000), el cual es 1 cuando hay total coincidencia entre los observadores. En psicología es difícil obtener el total de coincidencia, por lo cual se acepta el valor de Kappa de Cohen a partir de 0.61 (Abraira, 2001). Posteriormente, se calculó la Kappa de Cohen en intraobservador. En cada caso se trabajó con un nivel de confianza del 95\%.

\section{RESULTADOS}

En este apartado presentamos el registro elaborado, el cual está compuesto por la ficha técnica del registro (ver apéndice Figura 1), para contextualizar las características y elementos principales del instrumento. Posteriormente, se presenta el registro elaborado, Registro de Observación de Análisis del Movimiento (ROAM) (ver apéndice Figuras 2.1. y 2.2.), y después se muestra una tabla resumen del glosario del ROAM, para facilitar a los profesionales o investigadores su futura aplicación (ver apéndice Tabla 3).

\section{Validez de contenido: Técnica de jueces expertos}

Una vez aplicada la técnica de jueces expertos y después de cuatro versiones anteriores del registro, en la Tabla 4 (ver apéndice) se muestran algunas de las aportaciones hechas por los jueces durante la revisión de las distintas versiones del ROAM.

En la Tabla 5 (ver apéndice) se muestran las conclusiones finales de la última revisión del ROAM por parte de los expertos. 


\section{Fiabilidad: Índice de Kappa de Cohen}

La fiabilidad alcanzada por los observadores ha obtenido un índice de 0.72 , considerando la puntuación como buena, según refieren Landis y Koch (1977), Hoehler (2000) y Abraira (2001) (ver Tabla 6). El ROAM fue puntuado por dos observadores distintos para determinar si los observadores habían visto lo mismo.

Tabla 6

Kappa de Cohen de la aplicación piloto del ROAM

\begin{tabular}{|c|c|c|c|c|c|c|}
\hline $\begin{array}{l}\text { Dimensiones } \\
\text { del ROAM }\end{array}$ & Cuerpo & Espacio & Dinámica & Relaciones & Acción & $\begin{array}{c}\text { Puntuación } \\
\text { total }\end{array}$ \\
\hline $\begin{array}{l}\text { Kappa inter- } \\
\text { observadores* }\end{array}$ & 0.85 & 0.88 & 0.83 & 0.61 & 0.77 & 0.72 \\
\hline $\begin{array}{l}\text { Fuerza de } \\
\text { concordancia }\end{array}$ & MB & MB & MB & $B$ & B & B \\
\hline $\begin{array}{l}\text { Kappa } 1 \text { intra- } \\
\text { observador** }\end{array}$ & 0.77 & 0.83 & 0.83 & 0.62 & 0.89 & 0.72 \\
\hline $\begin{array}{l}\text { Fuerza de } \\
\text { concordancia }\end{array}$ & B & MB & MB & B & MB & B \\
\hline $\begin{array}{l}\text { Kappa } 2 \text { intra- } \\
\text { observador }\end{array}$ & 0.71 & 0.94 & 0.94 & 0.71 & 0.81 & 0.77 \\
\hline $\begin{array}{l}\text { Fuerza de } \\
\text { concordancia }\end{array}$ & B & MB & MB & B & MB & $B$ \\
\hline
\end{tabular}

Nota: $\mathrm{MB}=$ Muy Buena, $\mathrm{B}=$ Buena, ${ }^{*}$ corresponde a la sesión 4 y $16,{ }^{* *}$ corresponde al observador $1,{ }^{* * *}$ corresponde al observador 2 . Fuente: elaboración propia.

\section{DISCUSIÓN}

En este artículo se describe el proceso de construcción de un registro de observación basado en la Teoría de Laban, pudiendo así analizar los patrones de movimiento de las personas. Laban postuló el AML, fundamentado en el estudio de la interacción e interrelación constante establecida entre la mente y el cuerpo y la intencionalidad de las acciones, como bases del estudio del movimiento humano y del registro de observación aquí presentado (Bishko, 2014).

El punto de partida del ROAM fue la determinación y definición de las variables disponibles, buscando la obtención de una herramienta útil para el análisis del movimiento en cualquier tipo de población (Laban, 1978). Además, es un tipo de análisis fundamentado en el lenguaje corporal, a diferencia de la mayoría de estudios donde predomina como foco de investigación el lenguaje oral (Anguera, 1989; Zhao, 2001).

De este modo, el registro creado, el ROAM, da información sobre las tendencias generales de los movimientos de la persona, teniendo en cuenta posibles limitaciones físicas, cognitivas, emocionales y relacionales, antes y después de una intervención en movimiento (Barnet-López, et al., 2015).

A lo largo del proceso de construcción del registro, fue necesario elaborar cuatro versiones de este, con el fin de obtener la aprobación unánime de todos los expertos. 
Posteriormente, la última versión del ROAM ha sido aplicada en su primer estudio exploratorio en población con DI, considerando variables de conducta y comunicación no verbal sistematizadas y cuantitativas, donde se establecen una serie de categorías o dimensiones (Anguera, 1989), que aportan al instrumento precisión y objetividad. De este modo, la técnica de jueces expertos proporciona evidencias de que el contenido del instrumento es acorde a los elementos que el registro tiene como objetivo analizar.

El estudio exploratorio fue aplicado a personas con DI; por ello, los resultados obtenidos no se pueden generalizar a otro tipo de poblaciones. No obstante, es un instrumento que, en futuras líneas de investigación, podría ser aplicable para el análisis de la población general, así como en otro tipo de disciplinas que comporten movimiento, coordinación o equilibrio. La DMT da importancia al estudio del análisis del movimiento como mecanismo de observación e interpretación del movimiento de las personas (Stanton-Jones, 1992).

EI ROAM nos permite evaluar el movimiento a través de la grabación, por lo que el terapeuta solo estará pendiente de lo que ocurre en la sesión, siendo él, tal y como lo describe Queyquep (2008), parte activa del proceso y de la acción. El tener las sesiones registradas con cámara de video nos permite, como apunta Anguera (1989), volver a ver lo ocurrido sin limitaciones, y observarlo y analizarlo de forma detallada. Así pues, el video tiene permanencia en el tiempo y no existen límites en cuanto a tamaño o complejidad de la sesión. La filmación es una buena opción para precisar las puntuaciones del registro de más de un observador, lo cual nos permite contrastar el grado de acuerdo entre ellos (Anguera, 1989).

En la fase exploratoria, el índice total del registro en la Kappa de Cohen en el análisis interobservador puntuó 0.72 . Respecto a cada dimensión, se obtienen valores buenos, excepto en relaciones, la cual presenta un valor moderado (0.61), debido a que tiene más variables que, por tanto, dificultan su validación (Landis y Koch, 1977 y Hoehler, 2000). Y es que la Kappa de Cohen es la medida adecuada para conocer el acuerdo entre dos o más observadores (Muñiz, 1992).

En el caso de nuestro estudio, el ROAM se establece como un método para la observación, que cumple con el criterio de rigurosidad y objetividad (Bakeman, Gottman, Portal, Argilaga y Villaseñor, 1989). Los resultados obtenidos en el análisis de los datos apuntan que el ROAM es un instrumento útil para evaluar el movimiento de las personas y que permitirá conocer patrones comunes de su movimiento, aportando nuevas líneas de investigación dentro del campo de la expresión no verbal. Este registro nos permite conocer la combinación de calidades de movimiento que puede realizar una persona y su intensidad y fluidez (Schinca, 2010). Así pues, integra los elementos más relevantes de la teoría del AML (Laban, 1987; Zhao, 2001).

Por ser un instrumento nuevo, precisará de un entrenamiento por parte del observador, tanto en la estructura y forma de la hoja como en el AML. Es por eso que su uso se limita a la DMT o a expertos en Laban.

\section{Limitaciones y futuras líneas de investigación}

El estudio que presentamos se basa en una muestra reducida dado su carácter exploratorio, así como por las características de la población a la que se dirige (PérezTejero, 2009). Por este motivo, se propone en futuras investigaciones una ampliación 
de los participantes con el objetivo de obtener datos sobre la validez y fiabilidad del ROAM a gran escala, ya que hay una escasez de estudios grupales y con personas adultas con DI. Además, esto nos permitirá obtener mayor información sobre la dimensión relaciones, la cual presenta más variables que las otras dimensiones y una puntuación más ajustada en la fuerza de concordancia en la Kappa de Cohen (0.61).

EI ROAM permite conocer las características del movimiento de las personas con DI; por ello, consideramos poder utilizar el ROAM en futuros estudios para realizar observaciones pre y post intervenciones y así conocer los cambios de movimiento del individuo.

En relación con el trabajo con personas con DI y con la finalidad de ajustar el instrumento, proponemos la inclusión de algunos ítems más cercanos a las características de las personas con DI, como por ejemplo las estereotipias.

Por último, poder complementar la información obtenida a través del ROAM, con la de otro instrumento o prueba adecuada para la aplicación con DI, tal y como se propone en estudio de Barnet-Lopez et al. (2015).

\section{Conclusiones}

Se elaboró un registro de observación, el ROAM, con el fin de analizar las características del movimiento general de personas con DI. Debido a su sistematización en cuanto a categorías y a la identificación de elementos no verbales, es un instrumento que determina adecuadamente los elementos que evalúa. A través de la profundización a nivel teórico y de la técnica de jueces expertos, se obtuvo la aplicación de la herramienta (en un primer estudio exploratorio), en relación con la validez de contenido.

EI ROAM ofrece un sistema de categorización abierto y deja un espacio para observaciones complementarias del terapeuta 0 del investigador; esto permite incorporar todo tipo de comentarios, tomando en cuenta la complejidad que presenta el AML de cada una de las personas con DI. Por lo tanto, el ROAM nos ofrece la categorización y puntuación de las variables básicas para el AML, contemplando un espacio libre para la reflexión y la discusión. Además, demuestra unos buenos niveles de fiabilidad inter e intra observadores.

\section{Aplicaciones prácticas}

EI ROAM nos permite analizar las características del movimiento general de la persona, teniendo en cuenta posibles limitaciones a nivel cognitivo, emocional y físico que pueden presentar las personas con DI. Así, puede ayudar en el diagnóstico y en determinar necesidades de trabajo, tanto en el ámbito deportivo cómo clínico. Además, proporciona la opción de seguimiento con el fin de conocer los beneficios de las sesiones realizadas y los cambios de movimiento de la persona con DI, contribuyendo en la mejora de su bienestar emocional y físico.

\section{AGRADECIMIENTOS}

A la Beca de Recerca Blanquerna, concedida por la Fundación Blanquerna, Universidad Ramón Llull. A los profesores del Máster de Danza Movimiento Terapia de 
la Universidad Autónoma de Barcelona y a la Fundación Ramón Noguera, por su colaboración y apoyo, tanto a los participantes del estudio como al personal de la fundación.

\section{REFERENCIAS}

Abraira, V. (2001). El índice kappa. Semergen-Medicina de Familia, 27(5), 247-249. Doi: https://doi.org/10.1016/S1138-3593(01)73955-X

Amaro, M., Marrero, A., Valencia, M., Blanca, S. y Moynelo, H. (1996). Principios básicos de la bioética. Revista Cubana de Enfermería, 12(1). Recuperado de http://scielo.sld.cu/scielo.php?script=sci arttext\&pid=S0864-03191996000 $\underline{100006}$

Anguera, M. T. (1989). Metodología de la Observación en las Ciencias Humanas. Recuperado de http://www.laislalibros.com/libros/metodologia-de-observacionciencias-humanas-L0280000964/

Anguera, M. T. (1999). Observación en psicología clínica: aplicaciones. Recuperado de $\quad$ https://books.google.co.cr/books/about/Observaci\%C3\%B3n en psicolog\%C3\%ADa cl\%C3\%ADnica.html?id=Rq3Wf7-jDxsC\&redir esc=y

Anguera, M. T. y Hernández-Mendo, A. (2014). Metodología observacional y psicología del deporte: Estado de la cuestión. Revista de Psicología del Deporte, 23(1), 103-109. Recuperado de: http://www.rpd-online.com/article/view/ v23-n1-anguera-hernandez-mendo

Bakeman, R., Gottman, J. M., Portal, M., Argilaga, M. y Villaseñor, A. (1989). Observación de la interacción: introducción al análisis secuencial. Revista interuniversitaria de formación del profesorado, (7), 191-193. Recuperado de https://dialnet.unirioja.es/servlet/libro?codigo=25496

Barnet-López, S., Pérez-Testor, S., Arbonés-García, M. y Guerra-Balic, M. (2015). La danza en personas con discapacidad intelectual: Revisión sistemática e identificación de los elementos necesarios para evaluar cambios de movimiento. Ausart Journal for Research in Art., 3 (1), 213-227. Doi: https://doi.org/10.1387/ausart.14398

Bishko, L. (2014). Animation principles and Laban movement analysis: movement frameworks for creating empathic character performances. In Tanenbaum, J., ElNasr, M.S. y Nixon, M. (eds.). Nonverbal communication in virtual worlds (pp. 177-203). Pittsburgh, PA: ETC Press. Recuperado de: http://repository.cmu.edu/etcpress/14

Chace, M. (1953). Dance as an adjunctive therapy with hospitalized mental patients. Bulletin of the Menninger Clinic, 17, 219-225. Recuperado de https://www.ncbi.nlm.nih.gov/pubmed/13106594

Fischman, D. (2001). Danzaterapia: orígenes y fundamentos. Recuperado de: http://www.brecha.com.ar/ 
Foroud, A. y Whishaw, I. (2006). Changes in the kinematic structure and non-kinematic features of movements during skilled reaching after stroke: a Laban Movement Analysis in two case studies. Journal of Neuroscience Methods, 158 (1), 137149. Doi: https://doi.org/10.1016/j.jneumeth.2006.05.007

Hoehler, F.K. (2000). Bias and prevalence effects on kappa viewed in terms of sensitivity and specificity. Journal of Clinical Epidemiology, 53(5), 499-503. Doi. https://doi.org/10.1016/S0895-4356(99)00174-2

Hurley, A., Tomasulo, D. y Fadt, A. (1998). Individual and group psychotherapy: Approaches for persons with mental retardation and developmental disabilities. Journal of Developmental and Physical Disabilities , 10 (4). 365-386. Recuperado de https://link.springer.com/article/10.1023/A:1021806605662

Izquierdo, C. y Anguera, M. T. (2000). Hacia un alfabeto compartido en la codificación del movimiento corporal en estudios observacionales. Psicothema, 12(Supl.2), 311-314. Recuperado de: http://www.psicothema.com/psicothema. $a s p ? i d=572$

Laban, R. (1978). Danza educativa moderna. Buenos Aires: Paidós.

Laban, R. (1987). El Dominio del Movimiento. Madrid: Fundamentos.

Landis, J.R. y Koch, G.G. (1977). The measurement of observer agreement for categorical data. Biometrics, 33(1), 159-174. Doi: https://doi.org/10.2307/ $\underline{2529310}$

Longstaff, J. S. (2007). Overview of Laban movement analysis \& Laban notation. Recuperado de: http://www.laban-analyses.org/laban analysis reviews/laban analysis notation/overview/summary.htm

Moore, C. and Yamamoto, K. 1988. Beyond words: Movement observation and analysis. Recuperado de https://books.google.co.cr/books?id=cflTArEUZyQC\&hl $=$ es\&source $=$ gbs similarbooks

Muñiz, J. (1992). Teoría Clásica de los Test. Recuperado de https://books.google.co.cr/books/about/Teor\%C3\%ADa cl\%C3\%A1sica de los tests.html?id=NnkbPwAACAAJ\&redir esc $=y$

Paredes, D. (2010). Bienestar emocional y expresión conductual en las personas con discapacidad intelectual. Educación y Diversidad, 4(2), 51-60. Recuperado de: https://dialnet.unirioja.es/servlet/articulo?codigo=3276331

Pérez-Tejero, J. (2009). La investigación en actividades físicas y deportes adaptados: un camino aún por recorrer. RICYDE. Revista Internacional de Ciencias del Deporte, 5(16), I-III. Recuperado de: http://www.cafyd.com/REVISTA/ojs/index.php/ricyde/article/view/247/161

Queyquep, E. (2008). Las teorías del movimiento de Laban: la perspectiva de una danzaterapeuta. En Wengrower, H. y Chaiklin, S. (coords.). La vida es danza. El arte y la ciencia de la Danza Movimiento Terapia (pp. 239-258). Recuperado de https://goo.gl/BWigHN 
Real Decreto 1971/1999, de 23 de Diciembre, de procedimiento para el reconocimiento, declaración y calificación del grado de minusvalía, BOE núm. 22. 3317-3410 (2000). Recuperado de: https://www.boe.es/buscar/doc.php?id= BOE-A-2000-1546

Ridocci, M. (2009). Expresión Corporal. Arte del movimiento: las bases prácticas del lenguaje expresivo. Madrid: Biblioteca Nueva. Recuperado de: https://www.agapea.com/libros/Expresion-Corporal-Arte-del-movimiento-Lasbases-practicas-del-lenguaje-expresivo-9788497429207-i.htm

Ros, A. (2009). Laban Movement Analysis. Una herramienta para la teoría y la práctica del movimiento. Estudis escènics: quaderns de l'Institut del Teatre. 35, 350-357. Recuperado de: http://www.raco.cat/index.php/EstudisEscenics/article/view/ $\underline{252853 / 339591}$

Sarriá, E. y Brioso, A. (1999). Categorización y Observación de las funciones, morfología y características espacio-temporales de la comunicación intencional preverbal. En Arguilaga, M. Observación de conducta interactiva en contextos naturales: Aplicaciones. Recuperado de https://goo.gl/xWPCnQ

Schinca, M. (2010). Expresión corporal: Técnica y expresión del movimiento. Madrid: Wolters Kluwer. Recuperado de: https://estudioschinca.com/Publicacion/Expresion-Corporal.html

Stanton-Jones, K. (1992). Dance Movement Therapy in Psychiatry. London: Routledge. Recuperado de http://trove.nla.gov.au/work/21088372?q\&versionld=25111782

Vella, G. y Torres, E. (2012). Desarrollo de un instrumento de observación en danza movimiento terapia (DMT). Papeles del Psicólogo, 33(2), 148-156. Recuperado de: https://dialnet.unirioja.es/servlet/articulo?codigo $=4455333$

Zhao, L. (2001). Synthesis and Acquisition of Laban Movement Analysis Qualitative Parameters for Communicative Gestures (Tesis doctoral. Universidad de Pennsylvania). Recuperado de: http://repository.upenn.edu/dissertations/ $\underline{\text { AAl3015399/ }}$

Participación: A- Financiamiento, B- Diseño del estudio, C- Recolección de datos, D- Análisis estadístico e interpretación de resultados, E- Preparación del manuscrito. 


\section{Apéndice}

\section{Ficha técnica}

Nombre: Registro de Observación de Análisis del Movimiento (ROAM)

Aplicación: el registro se aplica individualmente.

Duración: desde un minuto (mínimo) a una hora, aproximadamente; dependiendo de la actividad que se ha filmado.

Finalidad: evaluación general del movimiento de la persona.

Material: hoja de registro y lápiz para rellenarla.

Características básicas: el ROAM está compuesto por cinco dimensiones. Cada dimensión se evalúa de forma independiente, aportando, posteriormente, un conocimiento general al profesional del análisis del movimiento del paciente. Sus ámbitos de aplicación son el clínico y el investigador; está pensado para ser un instrumento de fácil aplicación. Proporciona una manera clara, rápida y efectiva para determinar los elementos generales de las cinco dimensiones de la estrella de Laban. Está dirigido a profesionales de la DMT o expertos en el AML; es decir, profesionales o investigadores familiarizados en el lenguaje de Laban.

Figura 1. Ficha Técnica del ROAM. Fuente: Elaboración propia. 


\section{REGISTRO DE OBSERVACIÓN DE ANÁLISIS DEL MOVIMIENTO (ROAM)}

Fundamentado en la Teoria de Rudolf Laban: La estrella de Laban
NOMBRE:
EDAD:
N SESIÓN:
DIAGNÓSTICO:

Observe cada una de las dimensiones del ROAM. Cada dimensión está conformada por diversos aspectos, escoja la opción predominante y rodéela. Escriba sus comentarios en Observaciones Complementarias (O.C.) en cada una de las dimensiones, si lo precisa.

1. DIMENSIÓN: CUERPO

\begin{tabular}{lccccc}
\hline \multicolumn{5}{c}{ ORGANIZACIÓN Y USO DEL CUERPO } \\
Inestable \\
\hline Enraizamiento & -2 & -1 & 0 & 1 & 2 \\
Equilibrio & -2 & -1 & 0 & 1 & 2 \\
\hline \multicolumn{5}{c}{ Menos } \\
\hline Superior & 1 & 2 & 3 & 4 & 5 \\
Inferior & 1 & 2 & 3 & 4 & 5 \\
\hline Derecha & 1 & 2 & 3 & 4 & 5 \\
Izquierda & 1 & 2 & 3 & 4 & 5 \\
\hline
\end{tabular}

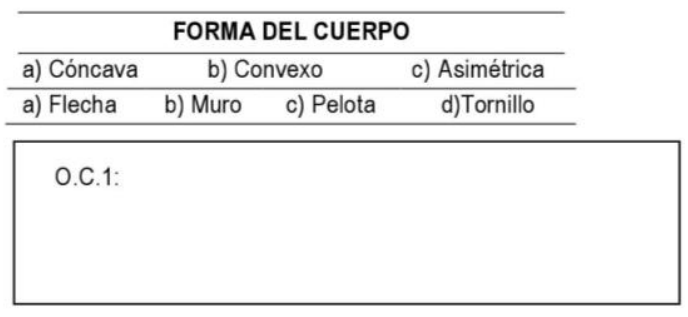

2. DIMENSIÓN: ESPACIO

\begin{tabular}{|c|c|c|c|c|c|}
\hline KINESFERA & \multicolumn{2}{|c|}{ Pequeña } & \multicolumn{2}{|c|}{ Mediana } & Grande \\
\hline Personal & 1 & 2 & 3 & 4 & 5 \\
\hline General & 1 & 2 & 3 & 4 & 5 \\
\hline \multirow[t]{2}{*}{ NIVEL } & Bajo & Medio-Bajo & Medio & Medio-Alto & Alto \\
\hline & 1 & 2 & 3 & 4 & 5 \\
\hline \multicolumn{2}{|c|}{$\begin{array}{l}\text { PLANOS } \\
{ }^{*} \text { Rodea una opción }\end{array}$} & \multicolumn{2}{|c|}{$\begin{array}{l}\text { Horizontal } \\
\text { (mesa) }\end{array}$} & $\begin{array}{l}\text { Sagital } \\
\text { (rueda) }\end{array}$ & $\begin{array}{l}\text { Vertical } \\
\text { (puerta) }\end{array}$ \\
\hline
\end{tabular}

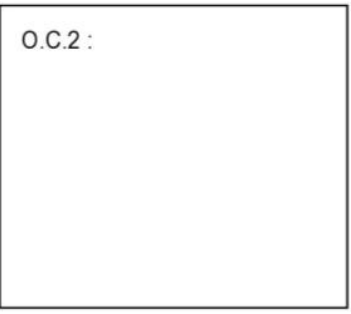

3. DIMENSIÓN: DINÁMICA

\begin{tabular}{|c|c|c|c|c|}
\hline $1=$ totalmente & $2=$ tendencia & $3=$ no destacan & $4=$ tendencia & $5=$ totalmente \\
\hline LIBRE $=1$ & 2 & $\begin{array}{c}\text { FLUJO } \\
3\end{array}$ & 4 & $5=$ CONDUCIDO \\
\hline INDIRECTO $=1$ & 2 & $\begin{array}{c}\text { ESPACIO } \\
3 \\
\end{array}$ & 4 & $\mathbf{5}=$ DIRECTO \\
\hline LIGERO $=1$ & 2 & $\begin{array}{c}\text { PESO } \\
3\end{array}$ & 4 & $\mathbf{5}=\mathrm{FIRME}$ \\
\hline SOSTENIDO = 1 & 2 & $\begin{array}{c}\text { TIEMPO } \\
3\end{array}$ & 4 & $5=$ SÚB BITO \\
\hline INDULGENTE & & & & LUCHA \\
\hline O.C.3 & & & & \\
\hline
\end{tabular}

Figura 2.1. Registro de Observación de Análisis del Movimiento (ROAM). Fuente: elaboración propia. 


\section{DIMENSIÓN: RELACIONES}
$1=$ nunca
2= rara vez
3= alguna vez
4= casi siempre
$\mathbf{5}=$ siempre

\begin{tabular}{|c|c|c|c|c|c|c|c|c|c|c|c|c|c|c|c|c|c|c|c|c|}
\hline \multirow[b]{3}{*}{ Iniciado } & \multicolumn{10}{|c|}{ CONTACTO VISUAL } & \multicolumn{10}{|c|}{ CONTACTO FISICO } \\
\hline & \multicolumn{5}{|c|}{ Terapeuta } & \multicolumn{5}{|c|}{ Grupo } & \multicolumn{5}{|c|}{ Terapeuta } & \multicolumn{5}{|c|}{ Grupo } \\
\hline & 1 & 2 & 3 & 4 & 5 & 1 & 2 & 3 & 4 & 5 & 1 & 2 & 3 & 4 & 5 & 1 & 2 & 3 & 4 & 5 \\
\hline Tolerado & 1 & 2 & 3 & 4 & 5 & 1 & 2 & 3 & 4 & 5 & 1 & 2 & 3 & 4 & 5 & 1 & 2 & 3 & 4 & 5 \\
\hline Recibido & 1 & 2 & 3 & 4 & 5 & 1 & 2 & 3 & 4 & 5 & 1 & 2 & 3 & 4 & 5 & 1 & 2 & 3 & 4 & 5 \\
\hline
\end{tabular}

COMUNICACIÓN NO VERBAL

\begin{tabular}{|c|c|c|c|c|c|c|c|c|c|c|}
\hline & \multicolumn{5}{|c|}{ Terapeuta } & \multicolumn{5}{|c|}{ Grupo } \\
\hline Se incluye en el círculo & 1 & 2 & 3 & 4 & 5 & 1 & 2 & 3 & 4 & 5 \\
\hline Inicia conductas hacia los demás & 1 & 2 & 3 & 4 & 5 & 1 & 2 & 3 & 4 & 5 \\
\hline Acepta propuestas en la interacción & 1 & 2 & 3 & 4 & 5 & 1 & 2 & 3 & 4 & 5 \\
\hline Toma la iniciativa & 1 & 2 & 3 & 4 & 5 & 1 & 2 & 3 & 4 & 5 \\
\hline Actitud de liderazgo & 1 & 2 & 3 & 4 & 5 & 1 & 2 & 3 & 4 & 5 \\
\hline Proximidad & 1 & 2 & 3 & 4 & 5 & 1 & 2 & 3 & 4 & 5 \\
\hline Muestra afecto por los demás & 1 & 2 & 3 & 4 & 5 & 1 & 2 & 3 & 4 & 5 \\
\hline Marca limites & 1 & 2 & 3 & 4 & 5 & 1 & 2 & 3 & 4 & 5 \\
\hline Sigue instrucciones & 1 & 2 & 3 & 4 & 5 & 1 & 2 & 3 & 4 & 5 \\
\hline Comparte los materiales & 1 & 2 & 3 & 4 & 5 & 1 & 2 & 3 & 4 & 5 \\
\hline
\end{tabular}

O.C. 4 :

\section{DIMENSIÓN: ACCIÓN}

$\mathbf{1 = n u n c a} \quad \mathbf{2}=$ rara vez $\quad 3=$ alguna vez $\quad \mathbf{4}=$ casi siempre $\quad \mathbf{5}=$ siempre

\begin{tabular}{llllllllllll}
\hline Presionar & 1 & 2 & 3 & 4 & 5 & Retorcerse & 1 & 2 & 3 & 4 & 5 \\
\hline Dar latigazos leves & 1 & 2 & 3 & 4 & 5 & Dar toques ligeros & 1 & 2 & 3 & 4 & 5 \\
\hline Dar puñetazos o arremeter & 1 & 2 & 3 & 4 & 5 & Hendir el aire & 1 & 2 & 3 & 4 & 5 \\
\hline Flotar o volar & 1 & 2 & 3 & 4 & 5 & Deslizarse & 1 & 2 & 3 & 4 & 5 \\
\hline
\end{tabular}

O.C. 5 :

Figura 2.2. Registro de Observación de Análisis del Movimiento (ROAM). Fuente: elaboración propia. 
Tabla 3

Resumen del glosario ROAM

1. DIMENSIÓN: Enraizamiento: conexión con la tierra y arraigamiento. Contacto con CUERPO la realidad.

Equilibrio: estabilidad del cuerpo con o sin movimiento.

Organización y usoCóncava: cuerpo curvado hacia delante, cabeza baja, retroversión de del cuerpohombros.

Convexa: cuerpo curvado hacia atrás, cabeza alta o flexión del cuello hacia arriba, antero-versión de hombros.

Asimétrica: posición corporal desigual.

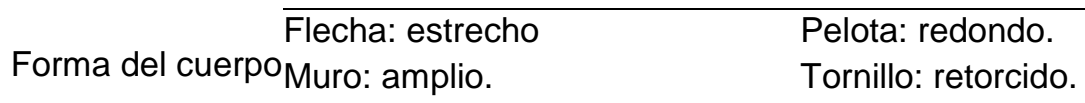
2. DIMENSIÓN: Kinesfera: espacio ocupado con las extremidades extendidas, ESPACIO imaginando al sujeto dentro de una esfera, alcanzando cualquiera de sus puntos.

KinesferaKinesfera personal: espacio Kinesfera general: espacio restante. dentro de la esfera.
Horizontal (mesa): divide el cuerpo y su movimiento de forma
Niveles y planos paralela al suelo. Movimientos de expansión y acercamiento. Sagital (rueda): divide el cuerpo y su movimiento de atrás hacia delante y viceversa.
Vertical (puerta): movimientos ascendentes y descendentes.
3. DIMENSIÓN: Flujo libre: acción difícil de Flujo conducido: acción que puede DINÁMICA detener. Más allá de los límites ser detenida y contenida dentro de corporales. los límites corporales.

Esfuerzos

Espacio indirecto: acción flexible, Espacio directo: acción foco cambiante o sin foco. concentrada, dirección en un solo punto.

Peso ligero: acción sin gravedad, Peso firme: acción con impacto menor presión. mayor la presión.

Tiempo sostenido: acción sin Tiempo súbito: acción rápida, prisa, persistente y duradera. urgente, fugaz.

\section{Continuación Tabla 3}

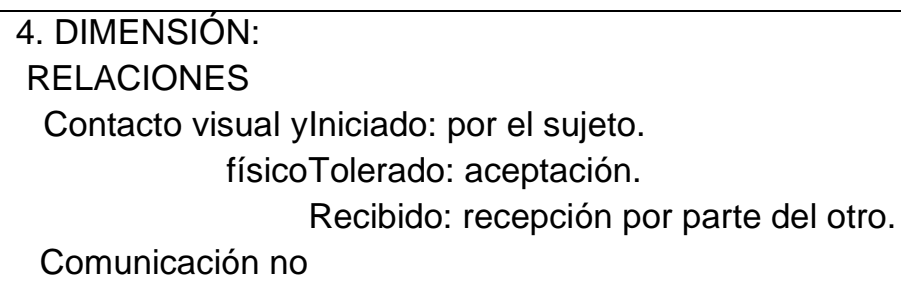


verbal

5. DIMENSIÓN: Las 8 acciones básicas: Ver tabla 1. ACCIÓN

Fuente: Elaboración propia.

Tabla 4

Resumen de las aportaciones de los jueces expertos durante las revisiones

Elementos a revisar o comentarios literales de los jueces

- Clarificación de la puntuación utilizada.

- Revisar el apartado Forma del cuerpo.

- Revisar los ítems referentes a aspectos relacionales e incluir algunos de nuevos: "puede seguir instrucciones, toma iniciativa...".

- Diferenciar entre contacto visual y físico.

- Añadir un espacio de "Observaciones complementarias" en cada dimensión.

- Organizar el registro según el modelo de la estrella de Laban.

- Centrar el registro en la expresión no verbal y en el movimiento.

Fuente: elaboración propia.

\section{Tabla 5}

$\underline{\text { Resumen de las aportaciones finales sobre la revisión de la última versión }}$

Aportaciones finales de los expertos

- El ROAM es algo nuevo que ayuda a comprender el AML; abre un nuevo modelo de comprensión para la investigación del AML.

- El ROAM abre camino, a nivel científico, en diversas disciplinas (DMT o danza).

- EI ROAM podría ser un instrumento adecuado para realizar el análisis del movimiento en muchos ámbitos diferentes; por ejemplo, en la práctica deportiva o a nivel terapéutico.

- Evaluar los esfuerzos de forma numérica aporta una nueva visión.

- El registro contempla la globalidad de las características principales del movimiento de la persona.

- Podrían incluir en la forma del cuerpo la opción de una postura erguida.

Fuente: elaboración propia. 